\title{
Individual differences in time estimation are associated with delay discounting and alcohol use
}

\author{
C. Henrico $\operatorname{Stam}^{1}$ (1) $\cdot$ Frederik M. van der Veen ${ }^{1} \cdot$ Ingmar H. A. Franken $^{1}$ \\ Published online: 3 July 2020 \\ (C) The Author(s) 2020
}

\begin{abstract}
Delay discounting is a commonly used behavioral measure of impulsive decision making and it has been shown that disturbed delay discounting is associated with drug dependence, problematic gambling, obesity and risk behavior. It is hypothesized that disturbed delay discounting may be due to aberrations in the subjective perception of time. In this study the associations were examined between subjective time estimation ability, impulsivity and substance use. A sample of healthy undergraduate students $(N=85)$ performed a time estimation task across 3 different intervals and completed a delay discounting questionnaire (MCQ). Substance use (alcohol and smoking) and personality characteristics reflecting impulsiveness (Eysenck-I7; BIS/BAS) were obtained via self-report. The results suggest that both delay discounting and degree of alcohol use are associated with time estimation abilities. There was a modest U-shaped association between delay discounting and one measure of time estimation (i.e., coefficient of variation). A higher, similar level of estimation error was found for both high and low delay discounting which can be seen as convergent evidence for the existence of a continuum of self-control, associated with behavioral risks and decisionmaking problems towards the extremes of the scale. Another measure of time estimation error (i.e., autocorrelation) was positively associated with alcohol use which implies a connection between time estimation and a risk factor for the development of alcohol use disorder. Findings suggest the existence of complex psychological associations between time estimation, impulsivity and addiction.
\end{abstract}

Keywords Impulsivity · Addiction · Time estimation · Delay discounting $\cdot$ Autocorrelation $\cdot$ BIS/BAS $\cdot$ Eysenck I7 $\cdot$ MCQ $\cdot$ Alcohol use

\section{Introduction}

It has been shown that trait impulsivity is closely linked to both addictive behavior (Verdejo-García et al. 2008; De Wit 2009; Stautz and Cooper 2013; Lee et al. 2019) and decision-

Electronic supplementary material The online version of this article (https://doi.org/10.1007/s12144-020-00899-7) contains supplementary material, which is available to authorized users.

C. Henrico Stam

c.h.stam@essb.eur.nl

Frederik M. van der Veen

veen@essb.eur.nl

Ingmar H. A. Franken

franken@essb.eur.nl

1 Department of Psychology, Education and Child Studies, Erasmus University, Rotterdam, The Netherlands making deficits (Franken et al. 2008). A commonly used behavioral measure of impulsive decision making is delay discounting which is known to be associated with addiction and other externalizing behavior (e.g. Bickel et al. 1999; Alessi and Petry 2003; Coffey et al. 2003; Reynolds 2006; MacKillop et al. 2011; Barlow et al. 2016; Amlung et al. 2017). Although the underlying mechanism of delay discounting is not completely understood (Odum 2011; Van den Bos and McClure 2013), one of the recurring hypotheses is that disturbed delay discounting may be due to aberrations in the subjective perception of time and/or magnitude of future consequences (e.g., Namboodiri et al. 2014b). A recent review (Paasche et al. 2019) has shown that a relationship between time perception and impulsivity in addictive disorders can be observed but is still largely underexplored.

Delay discounting is a common phenomenon. People in general 'discount' the value of delayed consequences relative to immediate consequences, but this tendency is more pronounced in impulsive individuals. Measures of delay discounting are based on the operational definition of a 
relative preference for smaller, more immediate rewards over larger, more delayed rewards (e.g. Rachlin et al. 1991; De Wit 2009). Empirically observed discount rates tend to decline when delay increases, which has resulted in a standard hyperbolic model to describe human and animals' intertemporal choice behavior. This is associated with the occurrence of reversal of preference which is seen as one of the hallmarks of impulsive behavior, whereby a decision maker switches choice from long term larger reward to short term smaller reward as time advances (Frederick et al. 2002). Discount rates as measured in the laboratory have been found to reliably predict behavior such as smoking and alcohol use (Bickel and Marsch 2001). There are many other self-reported impulsivity measurement instruments (e.g. Kirby and Finch 2010), for the present study the Eysenck I7 (Eysenck et al. 1985) and Behavioral Inhibition / Behavioral Activation Scales (BIS/ BAS; Carver and White 1994) were included to cover a broader measurement of trait impulsivity.

Explanations for delay discounting have been proposed and tested; most of these hypotheses state that the subjective perception of delay duration is a crucial variable (e.g. Gibbon 1977; Takahashi 2005, 2006; Takahashi et al. 2008; Zauberman et al. 2009; Kim and Zauberman 2009; Takahashi and Han 2013; Namboodiri et al. 2014a). Mean accuracy and Coefficient of Variation (CV) are common measures in time estimation (Grondin 2010). These measures are fundamental to Scalar Expectancy Theory (Gibbon 1977) which states that delay estimation is characterized by a (noisy) probability function of possible intervals stored in memory (Gallistel and Gibbon 2000) that is time-scale invariant. On this basis, it has been proposed that individual differences in $\mathrm{CV}$ may explain differences in delay discounting (Cui 2011). A less frequently studied measure is serial dependence which can be observed when successive time estimations are performed (Vroon 1976; Gilden et al. 1995; Wagenmakers et al. 2004). The extent to which people rely on their previous estimate(s) in a series can be measured by the parameters of an Autoregressive Integrated Moving Average model (ARIMA) or Autocorrelation Function (ACF). Importantly, it has been theorized that Autocorrelation of a person's biological clock may explain distortions such as hyperbolic discounting (Ray and Bossaerts 2011).

For experiments, it is important to consider the influence of duration of the interval that is being estimated as it has been shown that there are indications for different estimation processes underlying longer $(>3000 \mathrm{~ms}$, sometimes referred to as time estimation) and shorter intervals (sometimes referred to as time perception, Ulbrich et al. 2007). These different estimation processes for shorter and longer intervals could also lead to different associations between time estimation and delay discounting. From here on, this paper will refer to time estimation implying both short and long intervals.

In summary, there is a substantial amount of theory, clinical and experimental research available that suggests that impulsivity, and impulsivity-related psychiatric conditions such as addiction are associated with distortions in time perception. However, these relationships are still under-explored or undefined (Paasche et al. 2019). The time estimation ability measures considered in the present study are mean estimate, coefficient of variation and serial dependence.

\section{Hypotheses}

The main question for this study was whether time estimation performance and delay discounting are associated. It was expected that better time estimation performance would be associated with less delay discounting and that there could be a difference in association between delay discounting and shorter and longer intervals. As a second goal, the relationship between time estimation characteristics and behavioral measures of impulsivity was explored as measured by (a) selfassessed personality characteristics and (b) self-assessed substance use. To answer these questions, time estimation ability was measured in a convenience sample of undergraduate students and analyzed for associations with delay discounting. Further, associations between time estimation and other impulsivity measures were examined (BIS/BAS and Eysenck I7). Finally, the relationship between time estimation errors and degree of alcohol use was explored, as heavy drinking in late adolescence is a known risk factor for the development of alcohol use disorder in adulthood (e.g. Marshall 2014).

\section{Methods}

\section{Participants}

The study was conducted within a convenience sample of undergraduate university students. Whilst this can be seen as a limitation to generalization of results (e.g. Hanel and Vione 2016), it was expected that the homogeneity in the sample would serve to control for potentially influential differences in cognitive resources that are important to this study. The group of participants consisted of 92 Dutch speaking students recruited from different faculties of the Erasmus University (Social and behavioral studies, 73\%; Economics and management studies, 19\%, Other studies, $8 \%$ ). Participants were rewarded for their contribution by a payment of 10 euro in cash and undergraduate psychology students also received $1 \mathrm{~h}$ of course credit. In total there were 66 females and 26 male participants in the study.

\section{Apparatus/Instruments}

The experiment was conducted in two sessions. Each participant worked at a separate computer with headphones situated in a sound isolated cubicle in the Erasmus Behavioral Lab to 
minimize the risk of disturbance. Stimuli were presented, and responses were recorded on the computers using programs developed in E-Prime Studio 2.0 (version 2.0.10.252) for the time estimation task and Qualtrics (Provo, UT, USA, 2018) for other tasks.

\section{Materials and Procedure}

\section{Time Reproduction Task}

A combination of previous research has been followed for designing the task (Vroon 1976; Elbert et al. 1991; Wittmann et al. 2007). Temporal reproduction is one of the main methods to assess time estimation ability (Grondin 2010); this procedure involves presenting a target interval of a certain duration (e.g. auditory or visual) that participants are requested to reproduce subsequently. This method was chosen for the present study in a sequential paradigm (Vroon 1976), specifically as it allows to capture the necessary data to calculate mean, coefficient of variation and serial dependency measures (ARIMA and ACF) with a single task. Participants were instructed to reproduce the duration of standard tones $(3000 \mathrm{~ms}, 6000 \mathrm{~ms}$, and $9000 \mathrm{~ms})$ that were presented at $70 \mathrm{~dB}$ via headphones. The order of intervals was selected at random for each subject, leading to a total of 6 possible sequences. Participants were presented with the target interval and then asked to estimate each interval 30 times repeatedly, resulting in a total of 90 trials per subject. Each trial started with a $440 \mathrm{~Hz}$ tone presented two times for one of the target durations. Participants were instructed to reproduce the duration of the standard tone by pressing a key to switch off the tone when they believed the same duration had elapsed and were instructed not to count following Rattat and Droit-Volet (2012). The length of the interval was not disclosed to avoid comparisons and no feedback was provided on accuracy during the experiment to prevent learning effects. The fore period (i.e. the time between 'announcing' the stimulus and the actual stimulus) was fixed to $500 \mathrm{~ms}$ avoid the distortion observed by Mo and George (1977) and the maximum response time was set to $30,000 \mathrm{~ms}$. A progress status bar was updated on screen after each estimate to guide participant in understanding the degree of task completion; it was expected this would help maintain task focus. A schematic of the task is shown in Fig. 1.

\section{Delay Discounting Task}

In order to measure individual level of delay discounting, participants completed the Dutch version of the Monetary Choice Questionnaire (MCQ) which is one of the best validated discount measures (Kirby and Finch 2010). The questionnaire consists of 27 items where participants have to make a choice between a sooner, smaller reward and a larger, more distant reward (e.g. "Would you prefer (a) 78 euro today or (b) 80 euro in 162 days?"). Discount rates were estimated from the pattern of choices that participants made across the 27 questions. The outcome measure is the $\mathrm{k}$-value; this represents the individual discount rate by which participants devalue rewards based on delay. The higher the k-value, the more the individual will tend to exhibit impulsive behaviors. The answers to the questions were processed by means of an excel spreadsheet designed for this purpose (Kaplan et al. 2014). The geometric means of $\mathrm{k}$ were log-transformed as discount rates are positively skewed metrics (Kirby 2009).

\section{Self-Reported Impulsivity and Substance Use}

Participants completed a computerized versions of two questionnaires; the Dutch version of the Eysenck I7 (Lijffijt et al. 2005) and the Dutch version of the BIS/BAS (Franken et al. 2005). The Eysenck 17 scale has 54 items divided in three subscales: Impulsivity, Venturesomeness (Sensation seeking), and Empathy. The BIS/BAS scale has 20 items with four subscales: BAS (behavioral activation system) containing Drive, Fun seeking and Reward Responsiveness and BIS (behavioral inhibition system). Substance use was measured using the substance use questionnaire developed by Erasmus University, Department of Psychology, Education and Child Studies. This questionnaire has 68 items in total and is divided into three sections: smoking, alcohol and drugs (e.g. cocaine, cannabis, party drugs, heroin). Participants were asked to report on their use frequencies of these substances over time. The data obtained for smoking and alcohol was included in this study. For the analysis of alcohol use, the Quantity- Frequency-Variability index (QFV; Bongers et al. 1997; Lemmens et al. 1992) was used. Based on this QFV, participants were categorized either as light drinkers (the joined categories "none" and "light" or as heavy drinkers (i.e., the joined categories "medium" and "excessive" drinkers). Active smoking status was defined based on having smoked at least 10 cigarettes per day (Yi et al. 2016). Based on this criterium only two of the smoking participants qualified as active smoker. Therefore, smoking could not be investigated as part of this study due to insufficient presence in the sample.

The total experiment lasted $35 \mathrm{~min}$ on average. The order of the experiment was: time reproduction task, Eysenck I7, delay discounting task, BIS/BAS and substance use screener. Prior to the experiment, participants received a verbal (group) instruction and explanation from the researcher. Participants were asked for feedback after the experiment; 1 person reported error on the first interval of the time estimation task due to a misunderstanding. No other major issues were reported on procedure or materials.

\section{Statistical Analysis}

All statistical analyses were performed using SPSS (version 25), R Studio (version 1.1.463) and Microsoft Excel (version 16.22). For the time reproduction task, data was inspected for 
Fig. 1 Schematic of Time reproduction task. Note. Fore periods before trial start shown in $\mathrm{ms}$. After the first reference tone, there was a pause of $2000 \mathrm{~ms}$. After the trial announcement, there was a pause of $3000 \mathrm{~ms}$ before start. There was a pause of $500 \mathrm{~ms}$ after every trial estimate before starting the next

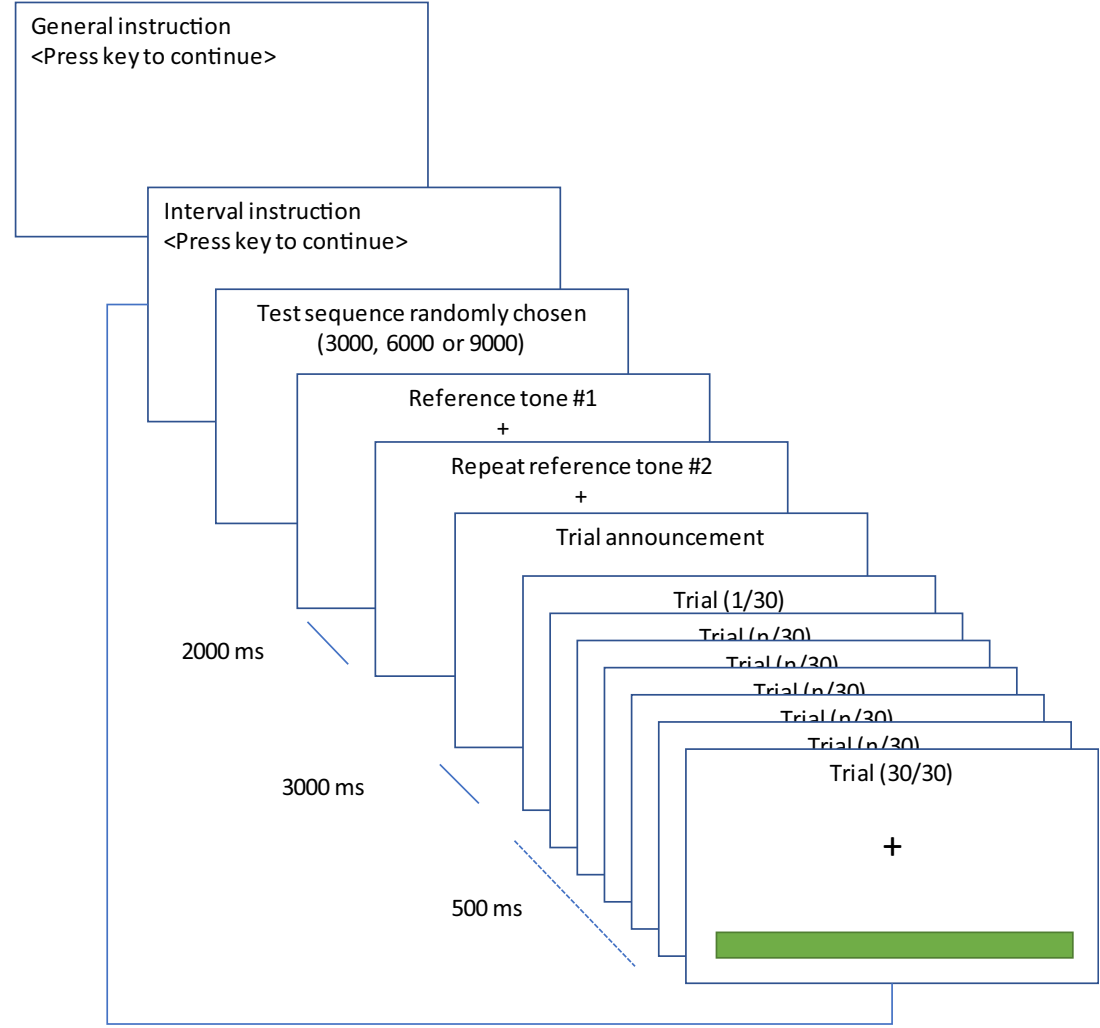

outliers following Ratcliff (1993). Cut-offs were set for very short ( $<300 \mathrm{~ms}$, most likely caused by premature responding) and very long responses $(>25,000 \mathrm{~ms}$, most likely due to lapses of concentration), similar for the three intervals. As a next step, at the subject level, responses outside a range of $2 S D$ from the mean were eliminated. These two steps removed $4.5 \%$ of responses. As a final step, group review (supported by box plot) identified 4 participants with significant mean outliers that were also excluded for further analysis (1 subject had completed the task incorrectly with majority of responses $<300$ ms cut-off on all intervals; 1 subject completed the first interval incorrectly and reported this to the experimenter; 2 participants had means outside a range of 2.5SD from group mean for multiple intervals.

The time estimation variables calculated in this study and the objective of each are summarized in Table 1.

Mean estimate and Coefficient of Variation were calculated and in addition, a recently proposed measure which is robust to drift (scaled Root Mean Squared Residuals; RMSR; Maaß and van Rijn 2018). Serial dependence was measured by calculating the individual parameters of an Autoregressive Integrated Moving Average model (ARIMA) and Autocorrelation Function (ACF) at subject/interval level. Autocorrelation estimates the relationships within a single variable (i.e. time estimation in the present study) that is measured in a regular series of events; the value can vary between -1.00 and 1.00 with a value of 0.00 indicating no dependency (Velicer and Fava 2003). The variables were analyzed with a
GLM for repeated measures to test for the presence of a potential trial sequence effect (Bausenhart et al. 2014), which could significantly influence the analysis results. Further details are provided in Supplementary Information.

The delay discounting task was generally completed in a coherent manner by participants. Overall consistency of choices was high $(M=97.9 \%, S D=2.7 \%)$ as measured by the excel tool (Kaplan et al. 2014). However, further analysis of the data (box plot) identified 3 participants with abnormally low discount rates compared to the group (virtually all responses on the delayed reward). As these are likely due to a misunderstanding of the task these participants were removed from the analysis.

Finally, the Shapiro-Wilk test of normality was used to investigate the effect of log transformation of the discount rate. The discount rate was found not to be normally distributed $(W=.967, p=.030)$ in the sample. This was unexpected. To address this problem for parametric statistical analyses, three groups reflecting the relative degree of delay discounting were defined post hoc (high, medium, low) using SPSS binning procedure, with cut-offs based on approximate equal weighting of percentiles (Table 2). Theoretically, this approach aligns with (low, medium, high) levels of the impulsive decision system (Bickel et al. 2011) that is proposed to be associated with risk of addiction. In experiments, similar segmentations of degrees of delay discounting have been followed (e.g. Yi et al. 2016; Cherniawsky and Holroyd 2013). 
Table 1 Summary of time estimation variables measured

\begin{tabular}{ll}
\hline Variable & Objective \\
\hline Mean & Mean biological clock speed \\
Coefficient of Variation (CV) & Variability (SD) in proportion to mean clock speed \\
Scaled Root mean squared residuals & Variability adjusted for drift proportional to mean \\
Autocorrelation & Degree of dependency on previous interval estimates \\
AR, MA coefficients of ARIMA model & Parameter modelling (autoregression and moving \\
& average) of a series of sequential estimations \\
\hline
\end{tabular}

\section{Results}

\section{Participant Characteristics}

A total of 85 participants were included in the analysis. Participant characteristics are shown in Table 3.

\section{Time Estimation Characteristics and Association with Delay Discounting}

Following pre-analysis (see Supplementary Information), the time estimation measures included in the behavioral part of the study were mean estimate, $\mathrm{CV}$ and ACF. Results of repeated measures GLM between time estimation characteristics and delay discounting groups are shown in Table 4.

After controlling for test sequence, the GLM with $\mathrm{CV}$ showed a main effect for delay discounting $(F(2,67)=$ $3.901, p=.025$ ), which indicates that the level of $\mathrm{CV}$ was different across the three delay discounting groups (low, medium and high DD). Differences in the absolute level of coefficient of variation for the three delay discounting groups are shown in Table 5 and Fig. 2.

Contrary to the hypothesis, the pattern that emerges in the Table 5 for the three intervals is U-shaped, i.e. the group of participants in medium DD has the lowest CV. This type of association does not correspond to the positive continuous relationship between $\mathrm{CV}$ and delay discounting that was expected based on theory; the mean CV for groups of low and high DD are quite similar across the three intervals (low DD $M=.154$; high DD $M=.158$ ), indicating that these groups show a similar time estimation error. However, in line with the hypothesis, High DD

Table 2 Categories of Delay discounting rates $(\log \mathrm{k})$

\begin{tabular}{llllll}
\hline Group & $n$ & Min. & Max. & $M$ & $S D$ \\
\hline Low DD & 29 & -8.752 & -5.987 & -6.437 & 0.601 \\
Medium DD & 31 & -5.977 & -4.151 & -5.012 & 0.566 \\
High DD & 25 & -4.144 & -2.290 & -3.576 & 0.560 \\
Total sample & & -8.752 & -2.290 & -5.076 & 1.278 \\
\hline
\end{tabular}

Note. $N=85$. Low/medium/high DD: low/medium/high delay discount rate group were found to have the highest $\mathrm{CV}$ on 2 out of the three intervals, but the association was strongest at the $3000 \mathrm{~ms}$ interval. This could be caused by the existence of different estimation processes for short and longer intervals (>3000 ms) that was expected. Contrary to the expectations, mean estimate and autocorrelation did not have a main effect with delay discounting. There were no significant interaction effects for the three variables, which is an indication that at the subject level, the time estimation characteristics for the three intervals $(3000,6000$ and $9000 \mathrm{~ms}$ ) within a delay discounting group (low, medium, high) were stable.

\section{Time Estimation and Association with Other Impulsivity Measures (BIS/BAS, Eysenck 17)}

For the BIS/BAS personality questionnaire, scores for BASReward Responsiveness, BAS-Drive and BAS-Fun Seeking

Table 3 Participant characteristics

\begin{tabular}{lr} 
Characteristic & Mean/Percentile (SD) \\
\hline Demographics & $20.66(4.32)$ \\
Age (years) & 71.8 \\
Gender (\% female) & \\
Behavioral measures & $-5.08(1.28)$ \\
Delay discount rate ln(k) & $.013(.019)$ \\
Delay discount rate (k) & \\
BIS/BAS & $21.99(2.44)$ \\
BIS & $10.59(1.61)$ \\
BAS Drive & $11.08(2.11)$ \\
BAS Fun seeking & $17.58(1.56)$ \\
BAS Reward responsiveness & \\
Eysenck I7 & $5.73(4.07)$ \\
Impulsivity & $9.28(3.74)$ \\
Sensation seeking & $14.88(2.52)$ \\
Empathy & \\
Self-reported substance use & 30.6 \\
Alcohol QFV (\% heavy drinkers) & $9.85(5.14)$ \\
Nr. of drinking days / month (heavy drinkers) & $6.60(2.21)$ \\
Drinks / drinking day (heavy drinkers) & \\
\hline Note. N = 85. Alcohol QFV: Alcohol Quantity/Frequency/Variability \\
score
\end{tabular}


Table 4 Time estimation characteristics and association with degree of delay discounting (low, medium, high)

\begin{tabular}{lcccllll}
\hline & \multicolumn{2}{l}{ Interval $-M,(S D)$} & & & General Linear model \\
\cline { 2 - 3 } & 1 & 2 & & & Main effect size & Interaction effect size $^{1}$ \\
\hline Mean estimate $\mathrm{ms}$ & $3048.5(1057.2)$ & $6144.6(2048.0)$ & $9771.4(3068.8)$ & & $F(2,82)=.901, p=.410$ & $F(4,164)=.638, p=.602$ \\
$\mathrm{CV}$ & $.136(.065)$ & $.141(.065)$ & $.161(.062)$ & & $F(2,67)=3.901, p=.025^{*}$ & $F(20,134)=1.102, p=.355$ \\
$\mathrm{ACF}$ & $.424(.226)$ & $.323(.238)$ & $.341(.227)$ & & $F(2,82)=.476, p=.623$ & $F(4,164)=1.492, p=.207$ \\
\hline
\end{tabular}

Note. $N=85$. CV: coefficient of variation; ACF: autocorrelation; Interval 1: $3000 \mathrm{~ms}$; Interval 2: $6000 \mathrm{~ms}$; Interval 3: $9000 \mathrm{~ms}$. *significant at $p<.05$.

${ }^{1}$ Huyn-Feldt correction applied

relationships were examined in relation to time estimation characteristics (mean estimate, coefficient of variation, autocorrelation). BAS-Drive was found to be associated with mean estimate, that is, both the main effect $(F(1,83)=4.657, p=.034)$ and interaction effect $(F(1.6,133.8)=3.941, p=.030)$ were significant. A simple linear regression analysis to predict mean estimate highlighted that participants higher in BAS-Drive demonstrated a faster 'biological' clock speed. Mean estimate decreased by -237.3 ms for each scale point increase of BAS Drive. Conversely, BIS was associated with longer mean estimates, nearing significance $(F(1,83)=3.428, p=.068)$. Taken together, these findings suggest that reinforcement sensitivity as measured by BIS/BAS is relevant in time estimation. Contrary to the expectations, other associations between time estimation and BAS subscales were not significant. No significant main effects or interaction effects of timing variables with I7-Impulsivity or I7Sensation seeking were found. Nearing significance was a main effect between mean estimate and Sensation seeking $(F(1,83)=$ $3.646, p=.060)$, where Sensation seeking was positively associated with shorter estimates. Overall, these results indicate that these self-reported measures of impulsivity were not strongly associated with time estimation ability.

\section{Time Estimation and Self-Reported Substance Use}

Results of repeated measures GLM between time estimation characteristics and severity of alcohol use are shown in Table 6.

For alcohol use, a significant main effect was found for autocorrelation $(F(1,83)=5.514, p=.021)$. Mean estimate

Table 5 Coefficient of variation (CV) segmented by degree of delay discounting by interval

\begin{tabular}{lllll}
\hline & \multicolumn{2}{l}{ CV for Interval - $M(S D)$} & \\
\cline { 2 - 4 } DD group & 1 & 2 & 3 & overall \\
\hline low DD $(n=29)$ & $.133(.062)$ & $.158(.080)$ & $.169(.060)$ & $.154(.053)$ \\
medium DD $(n=31)$ & $.121(.054)$ & $.124(.054)$ & $.143(.052)$ & $.129(.039)$ \\
high DD $(n=25)$ & $.158(.077)$ & $.142(.052)$ & $.173(.071)$ & $.158(.045)$
\end{tabular}

Note. $N=85 . \mathrm{CV}$ : coefficient of variation; Interval 1: $3000 \mathrm{~ms}$; Interval 2: $6000 \mathrm{~ms}$; Interval 3: $9000 \mathrm{~ms}$; DD: delay discounting group and CV main effects were not significant. No significant interaction effects (at the subject / interval level) were observed. Figure 3 shows the direction of the association between alcohol use profile and autocorrelation.

In line with the hypothesis, autocorrelation was higher for heavy drinkers than for light drinkers at each of the three intervals, suggesting that there was a positive association between severity of alcohol use and serial dependency that becomes more pronounced as the interval length increases. At $3000 \mathrm{~ms}$, the difference in ACF between heavy drinkers and light drinkers is relatively small, but the difference between the groups increases at $6000 \mathrm{~ms}$ and is largest at $9000 \mathrm{~ms}$. It was also observed that longer intervals (i.e. $6000 \mathrm{~ms}$ and $9000 \mathrm{~ms}$ ) are quite similar in ACF, which is another indication of different estimation processes for short and long intervals. Unexpectedly, no clear association was found between degree of delay discounting and alcohol use as measured by low or high QFV $(F(1,83=.175, p=.677)$ by using ANOVA-analysis.

\section{Discussion}

The main goal of this study was to examine the association between participants' time estimation characteristics and the degree of distortion in delay discounting. A modest, complex association was found between degree of delay discounting and the coefficient of variation measure of time estimation. As a second goal, associations between time estimation characteristics and behavioral measures of impulsivity were explored. It was found that autocorrelation of time estimation was considerably higher for heavy drinkers compared to light drinkers.

Contrary to the main hypothesis, no clear positive association was found between time estimation performance and the degree of delay discounting. Previous findings of a positive association between delay discounting and mean estimate (Baumann and Odum 2012) were not replicated. This inconsistent finding may have been caused by a difference in method: the present study's mean covers successive series of 30 estimates, whereas Baumann and Odum's mean was calculated based on a forced choice-model function of a participants' stimulus comparisons. Whilst it could be argued that a series' mean is susceptible to drift, this measure still captures a relevant 
Fig. 2 CV (estimated marginal means) by interval, by degree of delay discounting. Note. $\mathrm{N}=85$. $\mathrm{CV}$ : coefficient of variation; $\mathrm{DD}$ : delay discounting group; Interval 1: $3000 \mathrm{~ms}$; Interval 2: $6000 \mathrm{~ms}$; Interval 3: $9000 \mathrm{~ms}$. Error bars represent $95 \% \mathrm{CI}$

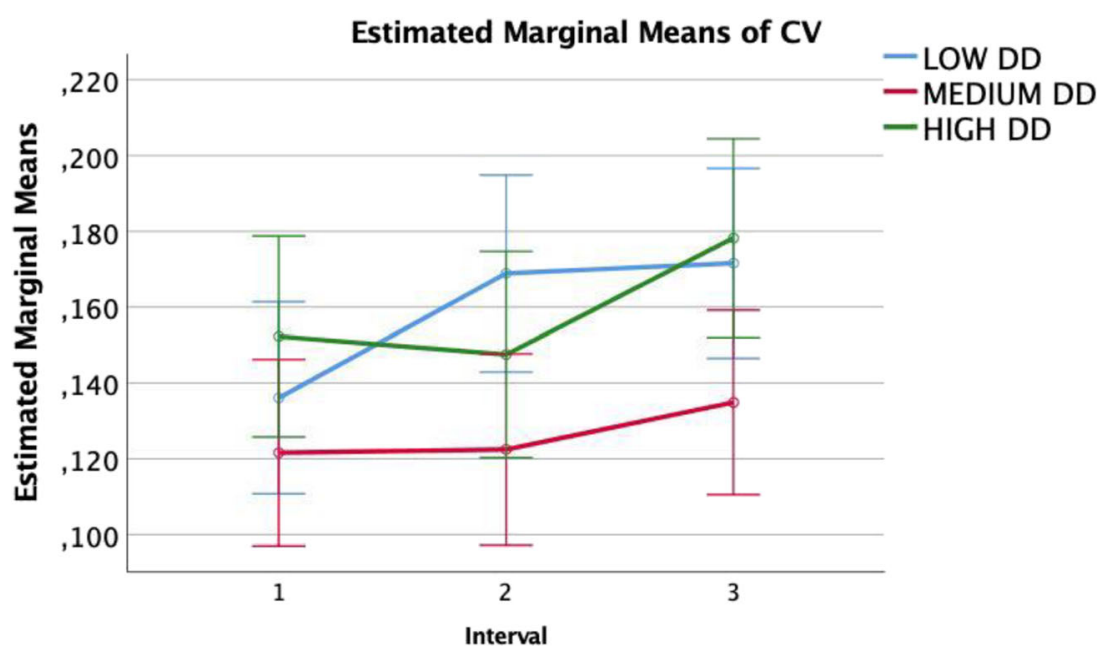

aspect of individual 'clock speed' given the high correlations for participants between intervals that were observed.

\section{Delay discounting's Complex Association with Time Estimation Ability}

A modest association was found between coefficient of variation of time estimation and delay discounting. This finding itself is consistent with proposed theory (Cui 2011), but the association is a complex U-shape, contrary to the linear pattern was expected. It was found that the medium delay discounting group had the lowest coefficient of variation, high and low delay discounting was characterized by higher, similar levels of coefficient of variation. There are two possible explanations for this. First, it has been argued that delay discounting, as a measure of self-control, lies on a continuum in which both extremes (i.e. high and low) are associated with psychopathology (Pinto et al. 2014). Where high delay discounting is perceived as an indication for low self-control/high impulsivity, low delay discounting was specifically found to be associated with psychopathology related to over-self-control/low impulsivity, such as Anorexia nervosa disorder, obsessive compulsive personality disorder and anxiety (Steinglass et al. 2017). The results in the present study can be interpreted as joining the high and low delay discounting groups on a similar type of time estimation error. Time estimation ability could therefore underly personality issues that are associated with both extremes of the delay discounting continuum, with the medium delay discounting group showing the best time estimation performance. Whilst this is a somewhat speculative claim, it has been shown that higher anxiety is associated with a subjective slower passage of time (Siegman 1962; Sarason and Stoops 1978). Second, the complex association found between delay discounting and coefficient of variation of time estimation can be interpreted as further support for the proposed existence of a U-shaped association between personality and time estimation stimulus complexity (Hogan 1978; Bachorowski and Newman 1985). In the present study, stimulus complexity was represented by the duration of the interval and personality by the degree of delay discounting. Although the shape of the identified association does not exactly follow theoretical predictions, it is an indication for the existence of a complex interplay between time estimation and dimensions of personality, such as degree of delay discounting.

\section{Limited Associations between Time Estimation and BIS/BAS and Eysenck I7}

As a second goal, the relationship between time estimation characteristics and other measures of impulsivity was explored. First, a significant association was found between mean estimate and BAS-Drive, where increases in BAS-Drive tend to lead to shorter estimates. This is consistent with previous findings (Corvi et al.
Table 6 Time estimation variables and association with alcohol use

\begin{tabular}{lll}
\hline & \multicolumn{2}{l}{ General linear model - Alcohol QFV } \\
\cline { 2 - 3 } & Main effect size & Interaction effect size $^{1}$ \\
\hline Mean estimate & $F(1,83)=.114, p=.737$ & $F(1.6,131.0)=1.568, p=.215$ \\
CV & $F(5,73)=.157, p=.977$ & $F(10,146)=.674, p=747$ \\
ACF & $F(1,83)=5.514, p=.021^{*}$ & $F(2.0,166.0)=.429, p=.652$ \\
\hline
\end{tabular}

$N=85$. CV: coefficient of variation; ACF: autocorrelation; Alcohol QFV: Alcohol Quantity/Frequency/ Variability score. *significant at $p<.05 .{ }^{1}$ Huyn-Feldt correction applied 
Fig. 3 Autocorrelation (estimated marginal means) by interval, for alcohol use groups. Note: Light drinkers $n=59$; heavy drinkers $n=26$ (measured by Alcohol Quantity/Frequency/Variability score); Interval 1: $3000 \mathrm{~ms}$; Interval 2: $6000 \mathrm{~ms}$; Interval 3: $9000 \mathrm{~ms}$. Error bars represent $95 \%$ CI
Estimated Marginal Means of Autocorrelation

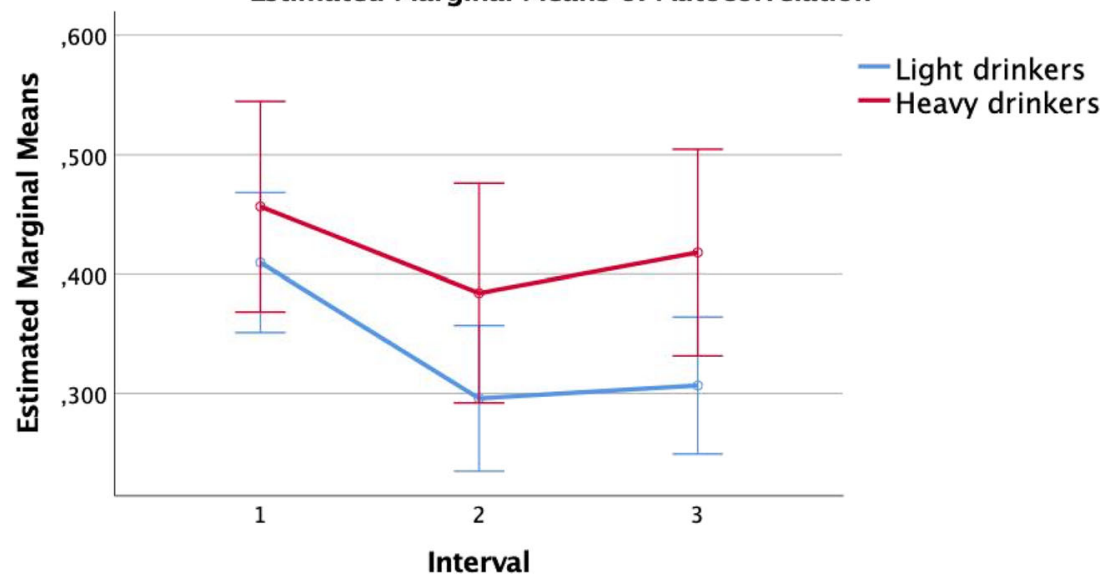

2012). This could be explained by a higher level of functional impulsivity that is associated with acting with less forethought (faster reaction times) while solving complex tasks when such a style is optimal (Dickman 1990; Avila 2001). Of the Eysenck scale, I7-Venturesomeness (Sensation seeking) was close to a significant association with mean estimate but I7-Impulsivity was unrelated; these findings are directionally consistent with prior research (Hodgins and Holub 2015). Coefficient of variation and autocorrelation of time estimation were not associated with BIS/BAS or Eysenck I7 scales.

\section{Associations between Time Estimation and Degree of Alcohol Use}

Finally, potential (direct) associations between time estimation characteristics and substance use were explored. It was found that autocorrelation of time estimation was positively associated with higher degree of alcohol use (QFV). This suggests that heavy drinkers have more difficulty with self-correction towards the original reference interval compared to light drinkers. This finding was consistent with theoretical predictions (Ray and Bossaerts 2011) that suggest that autocorrelation of the biological clock can explain distortions in delay discounting and impulsive behaviors such as addiction. A somewhat conflicting finding in the present study was that delay discounting as such was not directly associated with degree of alcohol use. However, this has also been reported in prior studies with clinical samples (e.g. Kirby and Petry 2004). This finding implies a connection between time estimation and a risk factor for the development of alcohol use disorder.

\section{Limitations}

This study has some limitations. Firstly, the study was conducted in a convenience sample of undergraduate students. Variation in delay discount rates is generally more pronounced when clinical groups are compared to healthy controls. This might explain why the associations identified were significant, but relatively modest. Therefore, replication of the study including a clinical sample will represent a relevant direction for further research. Secondly, it is unclear how stable the time estimation measures in this test will be over time. Prior research found a robust level of 1-year stability of time estimation measures (Anderson et al. 2014) which provides assurance, but this was less apparent at shorter intervals (10 s).

\section{Conclusions}

In conclusion, the findings suggest that delay discounting and potentially addictive behavior such as higher rates of alcohol use are associated with time estimation characteristics. For the main hypothesis, a modest, complex Ushaped association was found between degree of delay discounting and the coefficient of variation measure of time estimation. It appears that both high and low delay discounting groups were characterized by a similar level of time estimation error, whilst medium delay discounting was associated with the lowest level of error. The result can be seen as convergent evidence for the existence of a continuum of self-control/impulsivity with behavioral risks and problems on both extremes of the scale, which suggests that medium delay discounting could represent optimal decision making. These possible explanations require further research. For the exploratory objective, it was found that autocorrelation of time estimation was considerably higher for heavy drinkers compared to light drinkers. This can be understood as a form of persistence in error or a lower ability to self-detect and correct errors. In summary, these results provide further evidence for the existence of complex psychological associations between time estimation, addiction and impulsivity. Further research is required, focusing on replicating these findings including a clinical sample. 
Funding Information This research did not receive any specific grant from funding agencies in the public, commercial, or not-for-profit sectors.

\section{Compliance with Ethical Standards}

Conflict of Interest On behalf of all authors, the corresponding author states that there is no conflict of interest.

\section{Declarations of Interest none for all authors.}

Ethical Approval This study was performed in accordance with the local ethical guidelines of the Department of Psychology, Education and Child Studies, Erasmus University and with the 1964 Helsinki declaration and its later amendments.

Open Access This article is licensed under a Creative Commons Attribution 4.0 International License, which permits use, sharing, adaptation, distribution and reproduction in any medium or format, as long as you give appropriate credit to the original author(s) and the source, provide a link to the Creative Commons licence, and indicate if changes were made. The images or other third party material in this article are included in the article's Creative Commons licence, unless indicated otherwise in a credit line to the material. If material is not included in the article's Creative Commons licence and your intended use is not permitted by statutory regulation or exceeds the permitted use, you will need to obtain permission directly from the copyright holder. To view a copy of this licence, visit http://creativecommons.org/licenses/by/4.0/.

\section{References}

Alessi, S. M., \& Petry, N. M. (2003). Pathological gambling severity is associated with impulsivity in a delay discounting procedure. Behavioural Processes, 64(3), 345-354.

Amlung, M., Vedelago, L., Acker, J., Balodis, I., \& MacKillop, J. (2017). Steep delay discounting and addictive behavior: A meta-analysis of continuous associations. Addiction, 112(1), 51-62.

Anderson, J. W., Rueda, A., \& Schmitter-Edgecombe, M. (2014). The stability of time estimation in older adults. The International Journal of Aging and Human Development, 78(3), 259-276.

Avila, C. (2001). Distinguishing BIS-mediated and BAS-mediated disinhibition mechanisms: A comparison of disinhibition models of gray (1981, 1987) and of Patterson and Newman (1993). Journal of Personality and Social Psychology, 80(2), 311-324.

Bachorowski, J. A., \& Newman, J. P. (1985). Impulsivity in adults: Motor inhibition and time-interval estimation. Personality and Individual Differences, 6(1), 133-136.

Barlow, P., Reeves, A., McKee, M., Galea, G., \& Stuckler, D. (2016). Unhealthy diets, obesity and time discounting: A systematic literature review and network analysis. Obesity Reviews, 17(9), 810-819.

Baumann, A. A., \& Odum, A. L. (2012). Impulsivity, risk taking, and timing. Behavioural Processes, 90(3), 408-414.

Bausenhart, K. M., Dyjas, O., \& Ulrich, R. (2014). Temporal reproductions are influenced by an internal reference: Explaining the Vierordt effect. Acta Psychologica, 147, 60-67.

Bickel, W. K., \& Marsch, L. A. (2001). Toward a behavioral economic understanding of drug dependence: Delay discounting processes. Addiction, 96(1), 73-86.

Bickel, W. K., Odum, A. L., \& Madden, G. J. (1999). Impulsivity and cigarette smoking: Delay discounting in current, never, and exsmokers. Psychopharmacology, 146(4), 447-454.
Bickel, W. K., Jarmolowicz, D. P., Mueller, E. T., \& Gatchalian, K. M. (2011). The behavioral economics and neuroeconomics of reinforcer pathologies: Implications for etiology and treatment of addiction. Current Psychiatry Reports, 13(5), 406-415.

Bongers, I. M., van Oers, H. A., van de Goor, L. A., \& Garretsen, H. F. (1997). Alcohol use and problem drinking: Prevalences in the general Rotterdam population. Substance Use \& Misuse, 32(11), 14911512.

Carver, C. S., \& White, T. L. (1994). Behavioral inhibition, behavioral activation, and affective responses to impending reward and punishment: The BIS/BAS scales. Journal of Personality and Social Psychology, 67(2), 319-333.

Cherniawsky, A. S., \& Holroyd, C. B. (2013). High temporal discounters overvalue immediate rewards rather than undervalue future rewards: An event-related brain potential study. Cognitive, Affective, \& Behavioral Neuroscience, 13(1), 36-45.

Coffey, S. F., Gudleski, G. D., Saladin, M. E., \& Brady, K. T. (2003). Impulsivity and rapid discounting of delayed hypothetical rewards in cocaine-dependent individuals. Experimental and Clinical Psychopharmacology, 11(1), 18-25.

Corvi, A. P., Juergensen, J., Weaver, J. S., \& Demaree, H. A. (2012). Subjective time perception and behavioral activation system strength predict delay of gratification ability. Motivation and Emotion, 36(4), 483-490.

Cui, X. (2011). Hyperbolic discounting emerges from the scalar property of interval timing. Frontiers in Integrative Neuroscience, 5, 24.

De Wit, H. (2009). Impulsivity as a determinant and consequence of drug use: A review of underlying processes. Addiction Biology, 14(1), $22-31$.

Dickman, S. J. (1990). Functional and dysfunctional impulsivity: Personality and cognitive correlates. Journal of Personality and Social Psychology, 58(1), 95-102.

Elbert, T., Ulrich, R., Rockstroh, B., \& Lutzenberger, W. (1991). The processing of temporal intervals reflected by $\mathrm{CNV}$-like brain potentials. Psychophysiology, 28(6), 648-655.

Eysenck, S. B., Pearson, P. R., Easting, G., \& Allsopp, J. F. (1985). Age norms for impulsiveness, venturesomeness and empathy in adults. Personality and Individual Differences, 6(5), 613-619.

Franken, I. H., Muris, P., \& Rassin, E. (2005). Psychometric properties of the Dutch BIS/BAS scales. Journal of Psychopathology and Behavioral Assessment, 27(1), 25-30.

Franken, I. H., van Strien, J. W., Nijs, I., \& Muris, P. (2008). Impulsivity is associated with behavioral decision-making deficits. Psychiatry Research, 158(2), 155-163.

Frederick, S., Loewenstein, G., \& O'donoghue, T. (2002). Time discounting and time preference: A critical review. Journal of Economic Literature, 40(2), 351-401.

Gallistel, C. R., \& Gibbon, J. (2000). Time, rate, and conditioning. Psychological Review, 107(2), 289-344.

Gibbon, J. (1977). Scalar expectancy theory and Weber's law in animal timing. Psychological Review, 84(3), 279-325.

Gilden, D. L., Thornton, T., \& Mallon, M. W. (1995). 1/f noise in human cognition. Science, 267(5205), 1837-1839.

Grondin, S. (2010). Timing and time perception: A review of recent behavioral and neuroscience findings and theoretical directions. Attention, Perception, \& Psychophysics, 72(3), 561-582.

Hanel, P. H., \& Vione, K. C. (2016). Do student samples provide an accurate estimate of the general public? PLoS One, 11(12), e0168354.

Hodgins, D. C., \& Holub, A. (2015). Components of impulsivity in gambling disorder. International Journal of Mental Health and Addiction, 13(6), 699-711.

Hogan, H. W. (1978). A theoretical reconciliation of competing views of time perception. The American Journal of Psychology, 91, 417-428.

Kaplan, B. A., Lemley, S. M., Reed, D. D., \& Jarmolowicz, D. P. (2014). 21-and 27-item monetary choice questionnaire automated scorers. 
Kim, B. K., \& Zauberman, G. (2009). Perception of anticipatory time in temporal discounting. Journal of Neuroscience, Psychology, and Economics, 2(2), 91.

Kirby, K. N. (2009). One-year temporal stability of delay-discount rates. Psychonomic Bulletin \& Review, 16(3), 457-462.

Kirby, K. N., \& Finch, J. C. (2010). The hierarchical structure of selfreported impulsivity. Personality and Individual Differences, 48(6), 704-713.

Kirby, K. N., \& Petry, N. M. (2004). Heroin and cocaine abusers have higher discount rates for delayed rewards than alcoholics or nondrug-using controls. Addiction, 99(4), 461-471.

Lee, R. S., Hoppenbrouwers, S., \& Franken, I. (2019). A systematic metareview of impulsivity and compulsivity in addictive behaviors. Neuropsychology Review, 29(1), 14-26.

Lemmens, P. H. H. M., Tan, E. S., \& Knibbe, R. A. (1992). Measuring quantity and frequency of drinking in a general population survey: A comparison of five indices. Journal of Studies on Alcohol, 53(5), 476-486.

Lijffijt, M., Caci, H., \& Kenemans, J. L. (2005). Validation of the Dutch translation of the I7 questionnaire. Personality and Individual Differences, 38(5), 1123-1133.

Maaß, S. C., \& van Rijn, H. (2018). 1-s productions: A validation of an efficient measure of clock variability. Frontiers in Human Neuroscience, 12, 519 .

MacKillop, J., Amlung, M. T., Few, L. R., Ray, L. A., Sweet, L. H., \& Munafò, M. R. (2011). Delayed reward discounting and addictive behavior: A meta-analysis. Psychopharmacology, 216(3), 305-321.

Marshall, E. J. (2014). Adolescent alcohol use: Risks and consequences. Alcohol and Alcoholism, 49(2), 160-164.

Mo, S. S., \& George, E. J. (1977). Foreperiod effect on time estimation and simple reaction time. Acta Psychologica, 41(1), 47-59.

Namboodiri, K., Mohan, V., Mihalas, S., Marton, T., Shuler, H., \& Gilmer, M. (2014a). A general theory of intertemporal decisionmaking and the perception of time. Frontiers in Behavioral Neuroscience, 8, 61 .

Namboodiri, V. M. K., Mihalas, S., \& Hussain Shuler, M. G. (2014b). A temporal basis for Weber's law in value perception. Frontiers in Integrative Neuroscience, $8,79$.

Odum, A. L. (2011). Delay discounting: I'm ak, you're ak. Journal of the Experimental Analysis of Behavior, 96(3), 427-439.

Paasche, C., Weibel, S., Wittmann, M., \& Lalanne, L. (2019). Time perception and impulsivity: A proposed relationship in addictive disorders. Neuroscience \& Biobehavioral Reviews, 106, 182-201.

Pinto, A., Steinglass, J. E., Greene, A. L., Weber, E. U., \& Simpson, H. B. (2014). Capacity to delay reward differentiates obsessivecompulsive disorder and obsessive-compulsive personality disorder. Biological Psychiatry, 75(8), 653-659.

Rachlin, H., Raineri, A., \& Cross, D. (1991). Subjective probability and delay. Journal of the Experimental Analysis of Behavior, 55(2), 233-244.

Ratcliff, R. (1993). Methods for dealing with reaction time outliers. Psychological Bulletin, 114(3), 510-532.

Rattat, A. C., \& Droit-Volet, S. (2012). What is the best and easiest method of preventing counting in different temporal tasks? Behavior Research Methods, 44(1), 67-80.

Ray, D., \& Bossaerts, P. (2011). Positive temporal dependence of the biological clock implies hyperbolic discounting. Frontiers in Neuroscience, 5, 2.
Reynolds, B. (2006). A review of delay-discounting research with humans: Relations to drug use and gambling. Behavioural Pharmacology, 17(8), 651-667.

Sarason, I. G., \& Stoops, R. (1978). Test anxiety and the passage of time. Journal of Consulting and Clinical Psychology, 46(1), 102-109.

Siegman, A. W. (1962). Anxiety, impulse control, intelligence, and the estimation of time. Journal of Clinical Psychology, 18, 103-105.

Stautz, K., \& Cooper, A. (2013). Impulsivity-related personality traits and adolescent alcohol use: A meta-analytic review. Clinical Psychology Review, 33(4), 574-592.

Steinglass, J. E., Lempert, K. M., Choo, T. H., Kimeldorf, M. B., Wall, M., Walsh, B. T., Fyer, A. J., Schneier, F. R., \& Simpson, H. B. (2017). Temporal discounting across three psychiatric disorders: Anorexia nervosa, obsessive compulsive disorder, and social anxiety disorder. Depression and Anxiety, 34(5), 463-470.

Takahashi, T. (2005). Loss of self-control in intertemporal choice may be attributable to logarithmic time-perception. Medical Hypotheses, 65(4), 691-693.

Takahashi, T. (2006). Time-estimation error following Weber-Fechner law may explain subadditive time-discounting. Medical Hypotheses, 67(6), 1372-1374.

Takahashi, T., \& Han, R. (2013). Psychophysical neuroeconomics of decision making: Nonlinear time perception commonly explains anomalies in temporal and probability discounting. Applied Mathematics, 4(11), 1520-1525.

Takahashi, T., Oono, H., \& Radford, M. H. (2008). Psychophysics of time perception and intertemporal choice models. Physica A: Statistical Mechanics and its Applications, 387(8), 2066-2074.

Ulbrich, P., Churan, J., Fink, M., \& Wittmann, M. (2007). Temporal reproduction: Further evidence for two processes. Acta Psychologica, 125(1), 51-65.

Van den Bos, W., \& McClure, S. M. (2013). Towards a general model of temporal discounting. Journal of the Experimental Analysis of Behavior, 99(1), 58-73.

Velicer, W. F., \& Fava, J. L. (2003). Time series analysis. Handbook of Psychology, 581-606.

Verdejo-García, A., Lawrence, A. J., \& Clark, L. (2008). Impulsivity as a vulnerability marker for substance-use disorders: Review of findings from high-risk research, problem gamblers and genetic association studies. Neuroscience \& Biobehavioral Reviews, 32(4), 777-810.

Vroon, P. A. (1976). Sequential estimations of time. Acta Psychologica, 40(6), 475-487.

Wagenmakers, E. J., Farrell, S., \& Ratcliff, R. (2004). Estimation and interpretation of $1 / \mathrm{f} \alpha$ noise in human cognition. Psychonomic Bulletin \& Review, 11(4), 579-615.

Wittmann, M., Leland, D. S., Churan, J., \& Paulus, M. P. (2007). Impaired time perception and motor timing in stimulant-dependent subjects. Drug \& Alcohol Dependence, 90(2), 183-192.

Yi, R., Matusiewicz, A. K., \& Tyson, A. (2016). Delay discounting and preference reversals by cigarette smokers. The Psychological Record, 66(2), 235-242.

Zauberman, G., Kim, B. K., Malkoc, S. A., \& Bettman, J. R. (2009). Discounting time and time discounting: Subjective time perception and intertemporal preferences. Journal of Marketing Research, $46(4), 543-556$.

Publisher's Note Springer Nature remains neutral with regard to jurisdictional claims in published maps and institutional affiliations. 